brain. A computer can have a functional matrix of points stored at different addresses: the matrix does not need to be systematically arranged in space. Provided information is stored with each address to indicate its pictorial relation to other points, the information stored at the different addresses can be treated by the central processor as a picture. Kosslyn believes that the brain might function in a similar way. He puts his argument well and it should give pause to anyone who still believes that the operations that can be performed on visual images support the doctrine of isomorphism (that is the idea that each phenomenological dimension is represented by a corresponding physical dimension in the brain). It should, however, be noted that visual images could equally well depend upon the topological maps that are present in the primary visual areas: if so, they would involve some degree of isomorphism.

Kosslyn also rebuts those critics who have maintained that his results are produced entirely through subjects' expectations or by the experimental demands made on them. His experiment on stripes is enough to do this on its own, but for good measure he adds several others. For example, it has been argued that the rotation of mental images is a result of experience with objects rotating in the real world and that the speed at which a mental image is rotated depends on the subjects' expectations. Kosslyn shows that when subjects are required to rotate visual images of two different objects on a wheel, the speed of image rotation does not depend on the weight of the objects. Nevertheless, the subjects knew that in the physical world inertia would cause the heavier one at first to rotate more slowly than the lighter one, and could take this knowledge into account if required.

The least convincing part of Kosslyn's book is that dealing with the practical applications of his work. He suggests that it might be possible to discover which of the many routines he incorporates into his own program are poor in children who have deficient imagery: one could then concentrate on putting these routines right. More imaginatively, he proposes that the hallucinations experienced by schizophrenics are caused by deficiencies in the imaging process and might be corrected in the same way. The ideas are interesting but speculative: moreover, there is no evidence that people who claim to have no visual imagery suffer any intellectual loss. Nevertheless, I liked Kosslyn's tale of Nikola Tesla, the inventor of the AC generator, who claimed that having designed a machine he could run it in imagery for several weeks and then examine its parts to see how well they were wearing. It is a mercy he never applied his gifts to designing aero-engines.

Stuart Sutherland is Director of the Centre for Research on Perception and Cognition, University of Sussex.

\section{Another tool for the biochemist}

\section{Masamichi Tsuboi}

Applications of Infrared, Raman, and

Resonance Raman Spectroscopy in Biochemistry.

By Frank S. Parker.

Plenum: 1983. Pp.550. \$65, £50.05.

VIBRATIONAL spectroscopy (infrared and Raman spectroscopy) is not used by all biochemists. It is of little help, for example, in locating and measuring a newly found protein with a special biological activity. However, when the molecular conformation or conformational change of the new actor becomes the subject for study, vibrational spectroscopy is a powerful tool. For such tasks its use is extending steadily; we may dream that one day vibrational spectra of macromolecules will become the objects of routine analysis through a mathematical treatment, like X-ray diffractions or NMR spectra, after which we shall acquire a firm knowledge of the molecular force field with the assistance of $a b$ initio molecularorbital calculations. At present, however, the analysis is essentially empirical and requires the accumulation of great numbers of reference spectra. It also demands skill and experience on the part of the practitioners, a fact which seems to cause them no little pleasure.

In his new book, Frank S. Parker nicely surveys the present state of vibrational spectroscopy as applied to studies of biological macromolecules. The book opens with an attractive exposition of Fourier transform IR and other spectroscopic techniques and sampling methods. Dr Parker then deals with the applications of spectroscopy in a chapter on polypeptides and proteins, following this with descriptions of the spectra of eye tissues, purple membrane, enzymes, haemoproteins, metalloproteins, carbohydrates, nucleic acids and so on. Special emphasis is placed on the recent development of resonance Raman spectroscopy, which has brought great improvements in sensitivity and selectivity. Finally, Dr Parker covers the spectroscopic analysis of viruses and of biomembranes.

While the book is a review volume, covering much of the recent literature, it is written in a relaxed style which makes it much more pleasant reading than most of the papers on which it draws. The large number of illustrations -322 of them and the tables add a great deal further to the digestibility of the material. In organization, though, this is not a textbook. There is some repetition and choice of subject matter according to the author's preference; nevertheless graduate students in biology and chemistry will find it most instructive.Nor is it a handbook. For example the reference list at the end of each chapter is not intended to be exhaustive; but the information is well sign-posted and easily accessible. Above all, the work is permeated with Dr Parker's evident enthusiasm for the subject which makes his book unusually enjoyable.

Masamichi Tsuboi is a Professor of Physical Chemistry at the Faculty of Pharmaceutical Sciences, University of Tokyo.

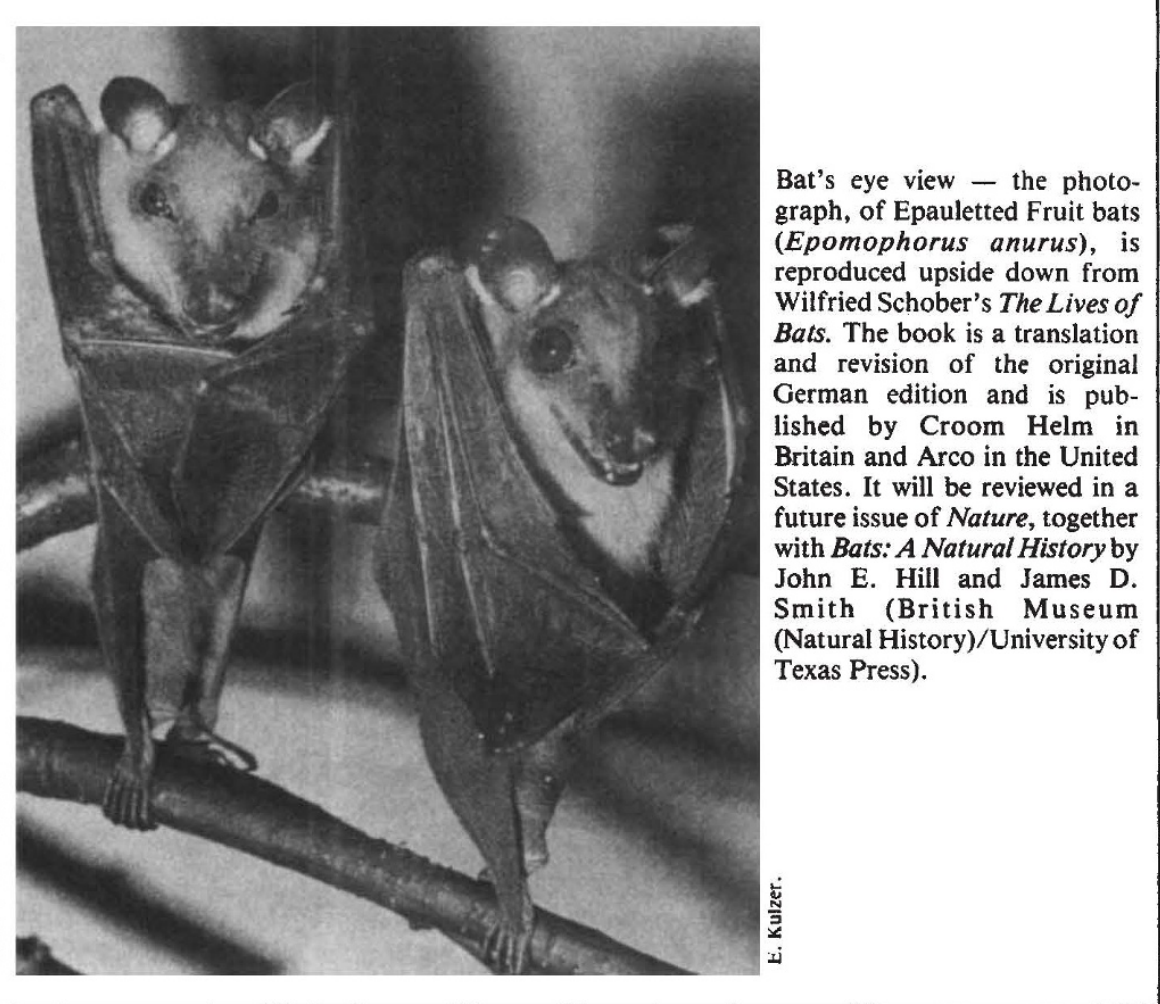

